\title{
Towards a Concept Inventory for Algorithm Analysis Topics
}

\author{
Mohammed F. Farghally*, Kyu Han Koh ${ }^{\dagger}$, Jeremy V. Ernst ${ }^{\ddagger}$, and Clifford A. Shaffer* \\ *mfseddik,shaffer@vt.edu, Department of Computer Science, Virginia Tech, Blacksburg, VA 24061 \\ ¥jvernst@vt.edu, School of Education, Virginia Tech, Blacksburg, VA 24061 \\ †kkoh@csustan.edu, Department of Computer Science, CSU-Stanislaus, Turlock, CA 95382
}

\begin{abstract}
We present initial results from our work towards developing a concept inventory for algorithm analysis (AACI) at the post-CS2 level. We used a Delphi process to identify a list of algorithm analysis topics that were considered both important and hard by surveying a panel of experienced instructors. Through a similar survey process, we identified a list of student misconceptions related to the identified topics. Based on this, a set of pilot AACI items were developed. We validated the misconceptions list by analyzing student responses to four administrations of the pilot AACI in two different universities during Fall 2015 and Spring 2016. Results revealed that a sufficient number of students held most of the misconceptions identified in the list.
\end{abstract}

\section{Keywords}

Algorithm Analysis; Concept Inventory; Misconceptions; Delphi Process

\section{INTRODUCTION}

Students start a given course with a set of preconceptions that can be leveraged to guide them to deeper understanding of specific concepts. In many cases, students hold misconceptions that, if untreated, can persist and hinder their understanding [21]. Before instructors can hope to develop curricular interventions to repair students' misconceptions, it is necessary to first identify them [13].

A Concept Inventory (CI) is a standardized assessment tool that evaluates whether a student's conceptual framework matches an accepted conceptual framework of a particular topic [8]. A CI can also be thought of as a set of Multiple Choice Questions (MCQs) in which each item targets one or more concepts, and item distractors correspond to student misconceptions related to these concepts [2].

Permission to make digital or hard copies of all or part of this work for personal or classroom use is granted without fee provided that copies are not made or distributed for profit or commercial advantage and that copies bear this notice and the full citation on the first page. Copyrights for components of this work owned by others than ACM must be honored. Abstracting with credit is permitted. To copy otherwise, or republish, to post on servers or to redistribute to lists, requires prior specific permission and/or a fee. Request permissions from permissions@ acm.org.

SIGCSE'17 March 8-11, 2017, Seatle, USA

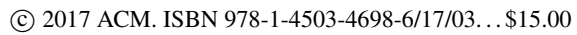

DOI: http://dx.doi.org/10.1145/3017680.3017756
The advent of CIs started with the Force Concept Inventory (FCI) [10]. The FCI was designed as a set of MCQs to probe students' understanding about the basic concepts of Newtonian mechanics. The FCI revealed a vast difference between student and expert understanding of core physics concepts, and it is now thought of as the gold standard that all other CIs are compared to [18].

CIs for Computer Science topics are only beginning to be created [18]. However, there is a large amount of ongoing work regarding the development of reliable and valid CIs for CS topics [20]. Some of these attempts were directed towards CS1 level courses like the introductory programming CI [2], the discrete mathematics CI [1], and the CS1 fundamentals CI [19]. Other efforts were directed towards higher level courses like the digital logic CI [8], the operating systems CI [21], the algorithms and data structures CI [4], and the computer architecture CI [16]. The interested reader can consult [18] for more information about CIs for CS-related topics.

To our knowledge, there has been no prior attempt to develop a CI for algorithm analysis topics. In [4], asymptotic runtime was defined as a fundamental topic to be included in an algorithms and data structures CI. However, no details were given for related misconceptions held by students, and no further topics were identified in the CI related to algorithm analysis. In [6], "order of growth" was defined as an important and difficult topic in discrete math. But no misconceptions were defined related to this topic.

This paper reports on three main contributions. First, we identified a list of important and difficult algorithm analysis concepts in a typical Data Structures and Algorithms (DSA) course taken at the post-CS2 level (we will refer to such a course as CS3). This list of topics was evaluated by a group of experienced instructors through a Delphi process. Second, we identified and validated the list of algorithm analysis misconceptions held by CS3 students. Third, we created a set of pilot AACI items focusing on the important and difficult concepts identified by the instructors. These pilot items probe student misconceptions from the identified misconceptions list.

We start by presenting results from the Delphi process in Section 2. Section 3 gives the initial list of misconceptions that we believe are held by CS3 students. Section 4 presents our pilot AACI items. Section 5 shows results from administering the AACI at two different universities. Section 6 presents future work plans. 


\section{FUNDAMENTAL CONCEPTS}

A CI is not intended to be a comprehensive examination on the topic [2]. A CI should mainly focus on those concepts identified to be difficult and important in a particular course [6]. To our knowledge, there is no prior listing of appropriate (i.e., difficult and important) algorithm analysis topics for a CS3-level course. Based on experience and the analysis of some DSA textbooks, we identified an initial list of potential topics as a starting point for evaluation.

To evaluate these concepts in terms of importance in the course and difficulty to students, we conducted a Delphi process [3] with a panel of experienced CS3 instructors. The goal was to provide ratings for the concepts from our initial list based on their importance and difficulty, along with suggestions for additional concepts to add. The Delphi process has been applied previously in developing several CIs to obtain a consensus among a group of experts through informed decisions $[6,13]$. The Delphi process has the advantage of overcoming bias that might exist for a single expert due to experience and specialty [13]. In addition, the Delphi process allows each person's views to be heard and provides the benefit of anonymous access to the views of other group members so that influence is only based on the logic of the arguments and not the experts' reputation [6].

Our Delphi panel was composed of ten experienced CS3 instructors from different institutions and countries (some from the US, others from Europe, and one from the Middle East), each with years of experience in teaching CS3-level courses. Some of them also have authored textbooks for DSA courses. We used a process with three main phases.

Phase 1: Initial Rating. We sent the experts (through email) our initial list of concepts along with a detailed explanation of what each concept means. We asked them to rate the concepts according to their perceived difficulty to their students and importance in the course. The ratings were from 0 to 10 , where 0 means not at all important or difficult, and 10 means very important or difficult. In addition, each expert was asked to add more concepts to our initial list if he believes that it should be added.

Phase 2: Negotiation. The difficulty and importance medians along with the Inter Quartile Ranges (IQRs) of each concept from Phase 1 were sent to the experts along with a modified list of concepts to reflect those concepts added by experts in Phase 1. Similarly, the experts were required to provide two ratings for each concept according to its difficulty and importance in the light of the results from Phase 1. In addition, experts were asked to provide written justifications if their ratings for a concept fell outside the IQR presented for that concept from Phase 1.

Phase 3: Final Rating. The experts were presented with the medians and IQRs from Phase 2 along with a list of anonymized justifications for ratings outside the IQR. Experts were asked to provide their final ratings in the light of the results from Phase 2 and the list of justifications. We found that only three experts changed their ratings a bit in this phase, in a way that did not change the overall medians and IQRs for any concept. Accordingly, we concluded with the results from Phase 2.

Table 1 shows the final concept ratings resulting from Phase 3 of the Delphi process. The medians provided by all ten experts are presented along with the IQRs.

Initially, we identified our Region Of Interest (ROI) as those concepts with importance and difficulty medians equal
Table 1: List of concepts with their rating medians and IQRs from the last phase of the Delphi process. The notation $x \mid y$ indicates the middle two from an even number of values. Suggested concepts by experts from Phase 1 are italicized. Concepts included in our ROI are emphasized.

\begin{tabular}{|c|c|c|}
\hline Concept & $\begin{array}{l}\text { Importance } \\
\text { median } \\
(\mathrm{IQR}) \\
\end{array}$ & $\begin{array}{l}\text { Difficulty } \\
\text { median } \\
(\mathrm{IQR})\end{array}$ \\
\hline \multicolumn{3}{|c|}{ Mathematical foundations } \\
\hline C1- Logs & $7 \mid 8(7-10)$ & $4(4-5)$ \\
\hline C2- Proofs by induction & $8(7-9)$ & $8(8-9)$ \\
\hline C3- Proofs by contradiction & $6(5-8)$ & $6 \mid 7(6-8)$ \\
\hline C4- Limits & $6(5-7)$ & $5(5-6)$ \\
\hline C5- Summations & $8(8-9)$ & $4(3-5)$ \\
\hline C6- Recurrence relations & $7(6-8)$ & $8(7-8)$ \\
\hline \multicolumn{3}{|c|}{ Growth rates } \\
\hline C7- Asymptotic growth & $10(9-10)$ & $6(4-8)$ \\
\hline C8- Inevitable crossing points & $7(6-8)$ & $5(5-6)$ \\
\hline C9- Effects of constants & $8(8-9)$ & $5(4-5)$ \\
\hline C10- Relative growth rates & $7 \mid 8(7-9)$ & $5(5-5)$ \\
\hline \multicolumn{3}{|c|}{ Concepts and notation for asymptotic running time analys } \\
\hline $\begin{array}{l}\text { C11- Upper bounds (Big- } \\
O)\end{array}$ & $10(9-10)$ & $7(6-9)$ \\
\hline C12- Lower Bounds (Big- $\Omega$ ) & $8 \mid 9(7-10)$ & $7 \mid 9(6-9)$ \\
\hline C13- Tight bounds (Big- $\Theta$ ) & $8(7-10)$ & $8(4-9)$ \\
\hline C14- Little o and $\omega$ & $4 \mid 5(2-6)$ & $5 \mid 6(5-8)$ \\
\hline $\begin{array}{l}\text { C15- Comparing functions via } \\
\text { limits }\end{array}$ & $6 \mid 7(6-7)$ & $6 \mid 7(5-8)$ \\
\hline \multicolumn{3}{|c|}{ Upper and lower bounds of problems } \\
\hline C16- Problem upper bound & $8(8-9)$ & $8(6-8)$ \\
\hline C17- problem lower bound & $8(7-9)$ & $8 \mid 9(7-9)$ \\
\hline $\begin{array}{l}\text { C18- Upper and lower } \\
\text { bounds of problems Vs. } \\
\text { algorithms }\end{array}$ & $7(7-8)$ & $8(7-9)$ \\
\hline \multicolumn{3}{|c|}{ Recursive analysis } \\
\hline $\begin{array}{l}\text { C19- Defining data structures } \\
\text { recursively }\end{array}$ & $7 \mid 8(6-9)$ & $7 \mid 8(8-9)$ \\
\hline $\begin{array}{l}\text { C20- Writing and analyzing re- } \\
\text { cursive functions }\end{array}$ & $9(7-10)$ & $9(8-9)$ \\
\hline \multicolumn{3}{|c|}{ Other topics } \\
\hline $\begin{array}{l}\text { C21- Best, average, and } \\
\text { worst cases }\end{array}$ & $9 \mid 10(9-10)$ & $7 \mid 8(6-9)$ \\
\hline $\begin{array}{l}\text { C22- Program efficiency } \\
\text { and analyzing loop con- } \\
\text { structs }\end{array}$ & $10(8-10)$ & $6(5-7)$ \\
\hline $\begin{array}{l}\text { C23- Analyzing space bounds } \\
\text { of algorithms }\end{array}$ & $8(7-9)$ & $6(5-8)$ \\
\hline $\begin{array}{l}\text { C24- Selecting an algorithm } \\
\text { based on a given use-case } \\
\text { scenario }\end{array}$ & $8 \mid 9(7-9)$ & $6 \mid 7(5-9)$ \\
\hline C25- Multi-parameter analysis & $5 \mid 7(5-7)$ & $6 \mid 7(6-8)$ \\
\hline C26- Space/time tradeoffs & $7 \mid 8(6-9)$ & $6 \mid 8(5-8)$ \\
\hline $\begin{array}{l}\text { C27- Empirical analysis and } \\
\text { code tuning }\end{array}$ & $4(4-7)$ & $7 \mid 8(5-8)$ \\
\hline C28- Amortized analysis & $6(6-8)$ & $8(7-9)$ \\
\hline C29- NP-Completeness & $6 \mid 7(5-8)$ & $10(9-10)$ \\
\hline
\end{tabular}

to or exceeding both the overall importance and difficulty medians of all the concepts. Our initial ROI included: C2, 
C11, C12, C13, C16, C17, C19, C20, C21, C24, and C26. In addition, we were also interested in four concepts close enough to the ROI, those representing C7, C10, C18, and $\mathrm{C} 22$. We were interested in those concepts because they are targeted by one or more identified misconceptions from our misconceptions list, as we will see in the next section.

However, addressing 15 concepts in a single CI is hard. We are restricted by time constraints when administering the AACI. Designing items for 15 concepts can result in a lengthy AACI that requires much time to administer. A typical CI should not require more than 30 minutes to complete without time pressure [1]. Accordingly, we decided to remove some concepts from our ROI. These concepts are C2, C19, and C20. C2 is related to proofs by induction. While this is an important mathematical prerequisite for a CS3-level course (and is a topic for a discrete math CI [1]), we believe that it is not an intrinsic concept in algorithm analysis. C19 and C20 are related to recursive analysis, and are also topics considered important to discrete math [1].

\section{IDENTIFYING MISCONCEPTIONS}

Previous studies on identifying misconceptions within CS have focused on programming [12], object oriented programming [11], operating systems [21], and digital logic [7, 9]. Little attention has so far been paid to misconceptions related to analyzing a problem structure and solution [4]. There is some research literature that tried to identify misconceptions related to algorithm efficiency. However, efficiency here does not mean the efficiency in an asymptotic sense (i.e., two algorithms may be in $O(n)$ but one algorithm is better than the other with a constant factor). For example, Ezer and Zur [5] conducted a study to reveal misconceptions in perceiving the efficiency of algorithms by high school students. This study revealed more basic misconceptions as follows. (Misconceptions are presented along with the concepts they are related to in parenthesis).

M1- Shorter programs are more efficient. (C22)

M2- Programs with less variables are more efficient. (C22)

M3- Programs having the same statements have the same efficiency even if the statements are in a different order. (C22)

M4- Two programs performing the same task are equally efficient. (C22)

A follow-up study [15] sought to determine if these four misconceptions are held by high school and early university students. The study revealed that both high school students and university students have the same misconceptions related to algorithm efficiency.

To our knowledge, the research literature does not identify student misconceptions related to asymptotic analysis of algorithms, upper bounds, lowers bounds, tight bounds, analyzing problems, and relative growth rates. However, a brief list of such misconceptions was described in the OpenDSA eTextbook [17]. These misconceptions were derived from more than 25 years of the author's experience teaching algorithm analysis for CS3 students. An adapted list of these misconceptions include:

M5- Students confuse the concept of upper bounds versus lower bounds. (C11)(C12) (C16)(C17)

M6- Students confuse the use of notations for upper bounds (Big-O), tight bounds (Big- $\Theta$ ), and lower bounds (Big$\Omega)$. (C11)(C12) (C13) (C16)(C17)
M7- Students confuse the concepts and notation of an upper bound on one hand versus worst case on the other hand. (C11)(C21)

M8- Students confuse the concepts and notation of a lower bound on one hand versus best case on the other hand. (C12) (C21)

M9- Students confuse the concepts and notation of a tight bound on one hand versus average case on the other hand. (C13) (C21)

M10- Students think that the best case of an algorithm will occur when the input size is as small as possible, and that the worst case will occur when the input size is as large as possible. (C21)

M11- Students confuse the upper and lower bounds of an algorithm on one hand versus the upper and lower bounds of a problem on the other. (C18)

Based on our own observations from a post-test analysis given to 53 students taking a CS3 course at a large research institution during Fall 2014, we have identified these additional misconceptions that we believe are held by some students.

M12- Students have poor intuition for the relative differences in common growth rates of algorithms. For example, they have poor intuition for the difference between $n^{2}$ versus $n \log n$ on the one hand and $n \log n$ versus $n$ on the other. $(\mathrm{C} 7)(\mathrm{C} 10)$

M13- Students have a weak grasp of logarithms. This expresses itself when students do not recognize that $n$ versus $\log n$ has the same relationship as $2^{n}$ versus $n$. (C7) (C10)

M14- Students confuse when to add and when to multiply when looking at loops for a function. This expresses itself when thinking that something costs $n$ ! (because they multiply $i$ from 1 to $n$ ) instead of $n^{2}$ (they should sum $i$ from 1 to $n)$. (C22)

M15- Loops always have cost that is linear on the maximum size of the control variable. That is, they assume the cost is always the same as when incrementing/decrementing the control variable by one. This is incorrect when, for example, the loop control variable is doubled or halved on each iteration. (C22)

M16- An algorithm with fewer loops is more efficient. (C22)

M17- Students confuse the best, average, and worst case scenarios of a particular algorithm. (C21)(C24)(C26)

As we see from the initial list of misconceptions, each concept from our ROI is covered at least once in this list. Accordingly, this list can be used as the basis for pilot AACI items. We asked our Delphi experts to provide feedback about our initial misconceptions list, since based on their long teaching experience, they should have an opinion regarding whether they feel each misconception is held by their students. Each expert was asked to categorize each misconception from our list according to whether it is important, and so should be retained in the list, or whether it should be removed. Results are summarized in Table 2.

From the table, we see that all misconceptions except M2 and M3 have at least 8 experts who rated them as important. For M2 and M3, 6 and 4 experts suggested the removal of these two misconceptions from the list, respectively. Before deciding to remove any misconception from the list, we have validated the list based on real student answers from 
Table 2: Expert feedback on list of misconceptions.

\begin{tabular}{ccc}
\hline & Important & Should be Removed \\
\hline M1 & 8 & 2 \\
\hline M2 & 4 & 6 \\
\hline M3 & 6 & 4 \\
\hline M4 & 9 & 1 \\
\hline M5 & 10 & 0 \\
\hline M6 & 10 & 0 \\
\hline M7 & 10 & 0 \\
\hline M8 & 10 & 0 \\
\hline M9 & 9 & 1 \\
\hline M10 & 8 & 2 \\
\hline M11 & 9 & 1 \\
\hline M12 & 10 & 0 \\
\hline M13 & 10 & 0 \\
\hline M14 & 9 & 1 \\
\hline M15 & 10 & 0 \\
\hline M16 & 8 & 2 \\
\hline M17 & 10 & 0 \\
\hline
\end{tabular}

four administrations of a pilot AACI. Results from these administrations are presented in Section 5 .

\section{PILOT AACI ITEMS}

Based on the identified list of fundamental concepts and the list of misconceptions, a set of pilot AACI items were created. These items are a mix of MCQs with justification, True and False with justification, and open ended questions. While items in most CIs are MCQs, open ended questions may be used to reveal other misconceptions that were not initially captured [2]. In addition, a student's justification for his answer to an MCQ or True and False question may be helpful as a supporting evidence that he is holding the misconception targeted by this question. Here we present some pilot AACI item examples.

- Provide the running time of the following code snippet. for $(i=1 ; i<=n ; i++)$ for $(j=1 ; j<=n ; j=j * 2)$

System.out.print $(i * j)$;

This item asks the student to write down the asymptotic (i.e., in terms of $\Theta$ ) running time of the presented code snippet. This is an example of an open ended item. A student having misconception M15 will provide an answer in $\Theta\left(n^{2}\right)$, ignoring that the second loop steps in multiples of 2 . In addition a student with misconception M14 will provide an answer in $\Theta(n !)$ or $\Theta\left(n^{n}\right)$.

- Mark all statements below about the upper, lower, and tight bounds of an algorithm as True or False. For any false statement, either correct it or explain why it is false.

(a) The upper bound of an algorithm is the growth rate that the algorithm has in its worst case.

(b) The lower bound of an algorithm is the growth rate that the algorithm has in its best case.

These two items test the ability to distinguish between the concepts of cases (best and worst) versus bounds (upper and lower). A student with misconception M7 will answer item (a) as True, as he is confused between the concepts of upper bound and worst case. A student with misconception M8 will answer item (b) as True, as he is confused between the concepts of lower bound and best case.

In a similar manner a list of 10 items was developed. Some of them are divided into sub-items, for a total of 29 items. Each item addresses one or more student misconception from our initial misconception list defined in Section 3.

The pilot AACI items were validated by our Delphi experts. We asked each expert to rate each item according to what extent he believes the item is good at addressing the required concept(s) and the corresponding student misconception(s). For all items, at least $80 \%$ of the experts rated them as good. Otherwise, they provided some rewording suggestions. All suggestions were taken into consideration before the pilot AACI administrations. Table 3 presents the concepts and misconceptions addressed by each pilot AACI item. The complete list of the pilot AACI items is available at [http://tinyurl.com/gtj62q9].

Table 3: Concepts and Misconceptions covered by each pilot AACI item.

\begin{tabular}{|c|c|c|}
\hline Item & Concept(s) & Misconception(s) \\
\hline Item 1 & $\mathrm{C} 22$ & $\mathrm{M} 1, \mathrm{M} 2, \mathrm{M} 4, \mathrm{M} 16$ \\
\hline Item 2 & $\mathrm{C} 22$ & M3, M4, M16 \\
\hline Item 3(a) & $\mathrm{C} 11$ & M7 \\
\hline Item 3(b) & $\mathrm{C} 12$ & M8 \\
\hline Item 3(c) & $\mathrm{C} 13$ & M5 \\
\hline Item $3(\mathrm{~d})$ & C11 & M5 \\
\hline Item $3(\mathrm{e})$ & $\mathrm{C} 12$ & M5 \\
\hline Item 3(f) & $\mathrm{C} 13$ & M9 \\
\hline Item $3(\mathrm{~g})$ & $\mathrm{C} 11, \mathrm{C} 12$ & $\mathrm{M} 7, \mathrm{M} 8$ \\
\hline Item 4(a) & $\mathrm{C} 21$ & M17 \\
\hline Item $4(\mathrm{~b})$ & $\mathrm{C} 21$ & M10 \\
\hline Item 4(c) & $\mathrm{C} 21$ & M10 \\
\hline Item $4(\mathrm{~d})$ & $\mathrm{C} 21$ & M17 \\
\hline Item 5(a) & $\mathrm{C} 16$ & M7 \\
\hline Item $5(\mathrm{~b})$ & $\mathrm{C} 17$ & M8 \\
\hline Item 5(c) & $\mathrm{C} 16, \mathrm{C} 17$ & M5 \\
\hline Item $5(\mathrm{~d})$ & C17, C18 & M11 \\
\hline Item $6(\mathrm{a})$ & $\mathrm{C} 22$ & M14, M15 \\
\hline Item $6(\mathrm{~b})$ & $\mathrm{C} 22$ & M14, M15 \\
\hline Item 6(c) & $\mathrm{C} 22$ & M14, M15 \\
\hline Item $7(\mathrm{a})$ & $\mathrm{C} 7, \mathrm{C} 10, \mathrm{C} 11$ & M6 \\
\hline Item $7(\mathrm{~b})$ & $\mathrm{C} 7, \mathrm{C} 10, \mathrm{C} 11$ & M6 \\
\hline Item $7(\mathrm{c})$ & $\mathrm{C} 7, \mathrm{C} 10, \mathrm{C} 12$ & M6 \\
\hline Item $7(\mathrm{~d})$ & $\mathrm{C} 7, \mathrm{C} 10, \mathrm{C} 13$ & M6 \\
\hline Item 8 & $\mathrm{C} 7, \mathrm{C} 10$ & M12, M13 \\
\hline Item $9(\mathrm{a})$ & $\mathrm{C} 7, \mathrm{C} 10, \mathrm{C} 11, \mathrm{C} 12$ & M6 \\
\hline Item $9(\mathrm{~b})$ & $\mathrm{C} 7, \mathrm{C} 10, \mathrm{C} 11, \mathrm{C} 12$ & M6 \\
\hline Item $9(\mathrm{c})$ & $\mathrm{C} 7, \mathrm{C} 10, \mathrm{C} 11, \mathrm{C} 12$ & M6 \\
\hline Item 10 & $\mathrm{C} 21, \mathrm{C} 24, \mathrm{C} 26$ & M17 \\
\hline
\end{tabular}

It is clear from the table that each concept from our ROI is covered in the pilot AACI at least once, as well as all of the misconceptions from our misconceptions list. There is no direct relationship between the difficulty or importance 
of a concept and the number of times it is addressed in the pilot items. What motivated the number of questions per concept are the number of misconceptions for that concept, and the number of items we believe a specific misconception can be revealed with.

\section{PILOT AACI ADMINISTRATION}

The pilot AACI was administered to 294 students in two different universities (a large research institution, and a teaching university) during Fall 2015 and Spring 2016. Selected students from both universities were enrolled in a CS3-level DSA course, and the pilot AACI was offered at the end of the course. Accordingly, students should have the algorithm analysis knowledge expected from a typical CS3 course including those fundamental topics identified in Section 2. In addition, they should also have pre-knowledge acquired from a pre-requisite CS2 course, in which they were exposed to the Big- $O$ notation, and the growth rates of simple mathematical functions. For both semesters in one university, the items were given as part of the final exam given at the end of the course. The overall time of the final was 2 hours, and we believe that most of the students have spent no more than 30-45 minutes answering the pilot AACI. At the other university, the students were given 30 minutes to solve the pilot AACI given as a post-test during the last week of the semester directly before their final exam.

Figure 1 shows results from the pilot AACI administrations in terms of how many students demonstrated a misconception in some answer within the four administrations. As we see from the figure, all misconceptions were detected except for M2. This misconception states that students think that a program with less variables is more efficient than one with more variables. This misconception was defined in [5] and [15] as a misconception held by high school and early university students. But we see no evidence from our pilot AACI that this misconception is held by CS3 students. This result coincides with what we found in Section 3, as $60 \%$ of the experts stated that this misconception should be removed from the list. [5] and [15] also defined M1, M3, and M4 as other misconceptions held by high school and early university students in algorithm efficiency topics. However, we found only small evidence that these misconceptions are held by CS3 students. Only $2 \%$ of the students among all four administrations expressed misconceptions M1 and M3 and $3 \%$ of the students expressed misconception M4. While these are not significant, still some CS3 students are holding these misconceptions. Based on this, we have decided to remove only M2 from our misconception list.

On the other hand, we see that a significant number of students provided answers supporting the remaining misconceptions. The two most prevalent misconceptions are M7 (68\%) and M8 (66\%). These are related to the students' confusion between the concepts of upper bounds versus worst cases on one hand and lower bounds versus best cases on the other hand. In addition, we see that $48 \%$ of the students demonstrated M9, which is related to confusion between the concepts of tight bound and average case. $59 \%$ of the students demonstrated M6, which is related to confusion in applying upper bounds, lower bounds, and tight bounds notations. This was revealed from students' answers to Items 7 and 9 related to relative growth rates. They used the incorrect notation to indicate the relationship between various growth rates. We also see that $49 \%$ of the students

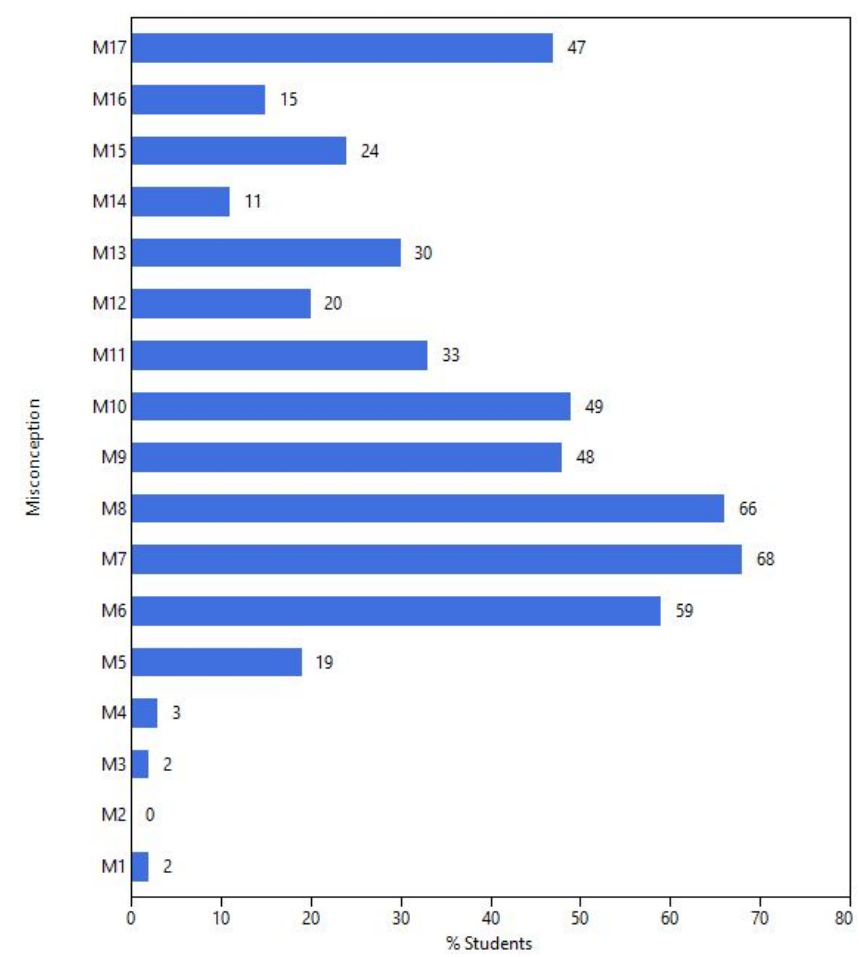

Figure 1: Results of the pilot AACI administrations

provided evidence (items 4(b) and 4(c)) for M10, related to the students' confusion about the effect of the size of the input on whether an algorithm can run in its best or worst cases. Students indicated that the best case of sequential search will occur when the array size is only a single element, while the worst case will occur when the array size is as large as possible. M17 is also significantly expressed in student answers (47\%). This misconception is related to students' confusion about the best, average, and worst cases of a specific algorithm. In items 4(a) and 4(d), students were confused between the best case and worst case of sequential search. In item 10, students were unable to determine the best sorting algorithm to use in a given use case scenario. However, it seems like students would have answered the questions correctly if they had memorized the running time of the algorithms in its best and worst cases. For example, in Item 10, if the students knew that the worst case running time of Heap sort is better than Quick sort and it requires less memory than Merge sort, then probably they would have answered the question correctly and the misconception wouldn't have been detected. Similar reasoning applies to Items 4(a) and 4(d). If the students knew that the best case for sequential search is when the target element is located in the first position of the array, and the worst case is when the target element is located at the end of the array or not found, then they probably would have answered the items correctly and M17 would not have been detected.

Based on this analysis, we believe that most of the misconceptions defined in the initial list are actually held by a significant proportion of CS3 students, as indicated from their responses to our pilot AACI. However, we believe that we need better items for misconception M17 not based on memorization to be able to test whether this misconception is actually held by CS3 students.

To test whether the pilot AACI is a reliable instrument 
in terms of internal consistency (i.e., all items measure the same construct), we computed Cronbach's-alpha coefficient using data from all four administrations. We found that the pilot AACI has a Cronbach's-alpha coefficient of 0.82 , which is good as stated in [14] for early research stages.

\section{CONCLUSIONS AND FUTURE WORK}

This paper described initial steps towards developing a CI for C3-level algorithm analysis topics. Our pilot AACI did a good job in revealing students' misconceptions expressed through their responses. For misconceptions M1 to M4, we didn't find enough evidence from students' responses, but still some students are holding these misconceptions. We removed M2 from the misconceptions list. In addition, we believe that better items are required to detect misconception M17. This misconception is related to the ability of students to differentiate between the best, average, and worst cases of algorithms.

The pilot AACI items have now received face validity by our Delphi experts, and they did a good job in detecting various students' misconceptions in initial testing. In addition, the pilot AACI was found to be a reliable instrument as indicated from a Cronbach's-alpha reliability coefficient of 0.82. Accordingly, the pilot AACI items can be used to develop further versions of the AACI. We plan to administer the prospective version of the AACI in several different universities to validate it and test its reliability.

\section{ACKNOWLEDGMENTS}

This work is supported by the National Science Foundation, under grants DUE-1139861 and IIS-1258471, and the VT-MENA program of Egypt.

\section{REFERENCES}

[1] V. L. Almstrum, P. B. Henderson, V. Harvey, C. Heeren, W. Marion, C. Riedesel, L.-K. Soh, and A. E. Tew. Concept inventories in computer science for the topic discrete mathematics. In ACM SIGCSE Bulletin, volume 38, pages 132-145.

[2] R. Caceffo, S. Wolfman, K. S. Booth, and R. Azevedo. Developing a computer science concept inventory for introductory programming. In Proceedings of the 47 th ACM Technical Symposium on Computing Science Education, pages 364-369.

[3] N. Dalkey and O. Helmer. An experimental application of the delphi method to the use of experts. Management science, 9(3):458-467, 1963.

[4] H. Danielsiek, W. Paul, and J. Vahrenhold. Detecting and understanding students' misconceptions related to algorithms and data structures. In Proceedings of the 43rd ACM technical symposium on Computer Science Education, pages 21-26.

[5] J. Gal-Ezer and E. Zur. The efficiency of algorithms misconceptions. Computers $\&$ Education, 42(3):215-226, 2004.

[6] K. Goldman, P. Gross, C. Heeren, G. Herman, L. Kaczmarczyk, M. C. Loui, and C. Zilles. Identifying important and difficult concepts in introductory computing courses using a delphi process. ACM SIGCSE Bulletin, 40(1):256-260.
[7] G. L. Herman, L. Kaczmarczyk, M. C. Loui, and C. Zilles. Proof by incomplete enumeration and other logical misconceptions. In Proceedings of the Fourth international Workshop on Computing Education Research, pages 59-70. ACM, 2008.

[8] G. L. Herman, M. C. Loui, and C. Zilles. Creating the digital logic concept inventory. In Proceedings of the 41st ACM technical symposium on Computer science education, pages 102-106.

[9] G. L. Herman, C. Zilles, and M. C. Loui. Work in progress-students' misconceptions about state in digital systems. In Frontiers in Education Conference, 2009. FIE'09. 39th IEEE, pages 1-2.

[10] D. Hestenes, M. Wells, G. Swackhamer, et al. Force concept inventory. The physics teacher, 30(3):141-158, 1992.

[11] S. Holland, R. Griffiths, and M. Woodman. Avoiding object misconceptions. In ACM SIGCSE Bulletin, volume 29, pages 131-134.

[12] L. C. Kaczmarczyk, E. R. Petrick, J. P. East, and G. L. Herman. Identifying student misconceptions of programming. In Proceedings of the 41st ACM technical symposium on Computer science education, pages 107-111.

[13] M. A. Nelson, M. R. Geist, R. L. Miller, R. A. Streveler, and B. M. Olds. How to create a concept inventory: The thermal and transport concept inventory. In Annual Conference of the American Educational Research Association, Chicago, IL, 2007.

[14] J. Nunnally. Psychometric theory, 2nd. edition. McGraw-Hill, New York, 1978.

[15] N. Özdener. A comparison of the misconceptions about the time-efficiency of algorithms by various profiles of computer-programming students. Computers \& Education, 51(3):1094-1102, 2008.

[16] L. Porter, S. Garcia, H.-W. Tseng, and D. Zingaro. Evaluating student understanding of core concepts in computer architecture. In Proceedings of the 18th ACM conference on Innovation and technology in computer science education, pages 279-284.

[17] C. A. Shaffer. OpenDSA CS3114 eTextbook. http://algoviz.org/OpenDSA/Books/CS3114/html/ AnalMisunderstanding.html, 2015.

[18] C. Taylor, D. Zingaro, L. Porter, K. Webb, C. Lee, and M. Clancy. Computer science concept inventories: past and future. Computer Science Education, 24(4):253-276, 2014.

[19] A. E. Tew and M. Guzdial. Developing a validated assessment of fundamental cs1 concepts. In Proceedings of the 41st ACM technical symposium on Computer science education, pages 97-101.

[20] A. E. Tew and M. Guzdial. The fcs1: a language independent assessment of cs1 knowledge. In Proceedings of the 42nd ACM technical symposium on Computer science education, pages 111-116.

[21] K. C. Webb and C. Taylor. Developing a pre-and post-course concept inventory to gauge operating systems learning. In Proceedings of the 45th ACM technical symposium on Computer science education, pages 103-108. 